\title{
Suppressor PAQR3 associated with the clinical significance and prognosis in esophageal squamous cell carcinoma
}

\author{
GE BAI $^{1 *}$, MEI YANG ${ }^{1 *}, \mathrm{CHAO}^{*} \mathrm{ZHENG}^{1}, \mathrm{LI}_{\mathrm{ZHANG}}{ }^{2}$ and MAYINUR ELI ${ }^{1}$ \\ ${ }^{1}$ Cancer Center, The First Affiliated Hospital of Xinjiang Medical University; ${ }^{2}$ VIP Medicine, The First Affiliated Hospital of \\ Xinjiang Medical University, Ürümqi, Xinjiang Uyghur Autonomous Region 830011, P.R. China
}

Received September 19, 2017; Accepted December 15, 2017

DOI: $10.3892 / \mathrm{ol} .2018 .8004$

\begin{abstract}
Progestin and adipoQ receptor family member 3 (PAQR3) is a novel tumor suppressor; however, its function in esophageal cancer is not well understood. The present study explored the association between PAQR3, and the survival and clinical phenotype in patients with esophageal squamous cell carcinoma (ESCC). The expression of PAQR3 in 80 cases of ESCC and its corresponding adjacent tissues was detected by reverse transcription-quantitative polymerase chain reaction. The results demonstrated that PAQR3 expression in cancer tissues was significantly lower compared with that in adjacent tissues. Clinicopathological analysis indicated that PAQR3 expression was significantly correlated with ethnicity $(\mathrm{P}=0.032)$, tumor length $(\mathrm{P}=0.019)$, lymph node metastasis $(\mathrm{P}=0.011)$ and local recurrence $(\mathrm{P}=0.009)$. Notably, the Kaplan-Meier survival curve demonstrated that a decrease in PAQR3 expression was associated with poor prognosis in patients with ESCC. Multivariate analysis indicated that PAQR3 expression was an independent prognostic indicator for patients with ESCC. PAQR3 may serve an important role in the progress of ESCC and become a potential candidate for ESCC targeted therapy.
\end{abstract}

Correspondence to: Professor Mayinur Eli, Cancer Center, The First Affiliated Hospital of Xinjiang Medical University, 137 Liyushan South Road, Ürümqi, Xinjiang Uyghur Autonomous Region 830011, P.R. China

E-mail: mayinur224@126.com

Professor Li Zhang, VIP Medicine, The First Affiliated Hospital of Xinjiang Medical University, 137 Liyushan South Road, Ürümqi, Xinjiang Uyghur Autonomous Region 830011, P.R. China

E-mail:263266418@qq.com

*Contributed equally

Key words: suppressor, extracellular signal-regulated kinase, prognosis, esophageal squamous cell carcinoma, progestin and adipoQ receptor family member 3

\section{Introduction}

Esophageal cancer (EC) is one of the most common types of malignancy globally, including esophageal squamous cell carcinoma (ESCC) and esophageal adenocarcinoma. In China, $>90 \%$ of ECs are ESCC (1). Currently, the morbidity of $\mathrm{EC}$ is increasing annually and the rate of metastasis remains high with a poor prognosis (2). The diagnosis of EC usually occurs in the middle and late stages due to the lack of symptoms in the early stage, and as a result, the 5-year survival of patients with EC is $18-30 \%$ (3). The RAS/rapidly accelerated fibrosarcoma (RAF)/extracellular signal-regulated kinase (ERK) signaling pathway is critical for the malignant biological behavior of ESCC (4). This pathway can be activated in multiple ways, and has an important role in the proliferation, metastasis and infiltration of ESCC (5). Therefore, the identification of molecules that specifically inhibit activation of the RAS/RAF/ERK signaling pathway may enable the development of novel treatments for ESCC.

Raf kinase trapping to Golgi apparatus [also known as progestin and adipoQ receptor family member 3 (PAQR3)] is a type III seven-transmembrane protein with an extracellular $\mathrm{N}$-terminus and a cytosolic $\mathrm{C}$-terminus determined in the membrane of the Golgi apparatus (6). Binding of PAQR3 to B-Raf and C-Raf kinases anchors cytosolic RAF to the Golgi apparatus, and prevents the binding of RAF to RAS (upstream) and MEK (downstream) (7). Blocking signal transduction from RAS inhibits the activation of the Ras/Raf/MEK/ERK signaling pathway, and consequently affects the proliferation and malignant transformation of cells, as well as the development and progression of cancer $(8,9)$.

Therefore, PAQR3 is considered a negative regulator that suppresses RAS/RAF/ERK pathway activation-dependent tumors $(10,11)$. A study by Wang et al (12) determined that kinases in the Raf/MEK/ERK signaling pathway were abnormally activated in various tissues of PAQR3 gene knockout mice. These knockout mice had significantly increased proliferation of epidermal keratinocytes, and PAQR3 gene deletion promoted the development and progression of dimethylbutylamine/12-Otetradecanoyl-phorbol-12-acetate-induced skin cancer. Other studies have reported downregulated expression of PAQR3 in liver (10), gastric $(13,14)$, colon $(15)$ and breast cancer (11), and determined that this downregulation was associated with tumor progression, metastasis and prognosis. However, the 
role and mechanism underlying the action of PAQR3 in ESCC has not been reported. Therefore, the purpose of the present study was to examine the association between PAQR 3 and the prognosis and clinicopathological characteristics of patients with ESCC, and to increase the understanding of the molecular functions of PAQR3.

\section{Materials and methods}

Ethical statement. All selected patients agreed to participate in the present study and signed informed consent forms. The present study was approved by the Ethics Committee of the First Affiliated Hospital of Xinjiang Medical University (Ürümqi, China), and was implemented in accordance with 'The Declaration of Helsinki'.

Tissue specimens. A total of 80 histopathological specimens from patients surgically diagnosed with ESCC between January and March 2012 were collected from the First Affiliated Hospital and Affiliated Tumor Hospital of Xinjiang Medical University. All patients had undergone radical surgery and had not received prior treatments. The clinicopathological data of these patients, including age, sex, ethnic group, tumor location, tumor length, tumor type, degree of differentiation, number of lymph node metastases and $\mathrm{T}$ staging, were obtained from the hospitals. A total of 40 male and 40 female patients (mean age, $54.7 \pm 12.6$ years) underwent tumor resection. Tumor and paracancerous histological normal tissue (PCHNT) specimens were obtained at the same time during surgery, and were stored at $-80^{\circ} \mathrm{C}$. PCHNT is defined as esophageal tissue that is located $\geq 5 \mathrm{~cm}$ away from the tumor margin and is confirmed to be normal esophageal tissue by postoperative histopathology. Patient follow-up was conducted from the day following surgery, and anastomotic or regional lymph node recurrence was diagnosed by clinical or pathological examination at 3 months post-operation. Local recurrence within 3 months following surgery was considered a part of the initial treatment process. Distant metastasis was defined as distant metastatic disease diagnosed by clinical examination or imaging. Disease-free survival (DFS) time was defined as the time from the day following surgery to the first recurrence or metastasis. Overall survival (OS) time was defined as the time from day 1 post-operation to last follow-up or mortality. Complete follow-up data included the time and location of recurrence or metastasis, and condition and time of survival. All patients were followed up until March 2017 and the duration of follow-up was 9-62 months (median, 28 months).

Cell culture. Human ESCC cell lines (ECA-109 and TE-1) were obtained from the Type Culture Collection of Chinese Academy of Sciences (Shanghai, China). They were maintained in Dulbecco's modified Eagle's medium (DMEM) containing $10 \%$ fetal bovine serum (both from Sigma-Aldrich; Merck KGaA, Darmstadt, Germany) at $37^{\circ} \mathrm{C}$ and in a $5 \% \mathrm{CO}_{2}$ atmosphere.

Plasmid and transfection. Full-length human PAQR3 cDNA was amplified using a polymerase chain reaction (PCR), as previously described (2). PCR products were purified and inserted into a pcDNA3.1(+) vector. The PAQR3-expressing plasmid (pcDNA3.1/PAQR3) or an empty vector were transfected into ECA-109 and TE-1 cells using Lipofectamine 2000 (Invitrogen; Thermo Fisher Scientific, Inc., Waltham, MA, USA), according to the manufacturer's instructions. A total of $24 \mathrm{~h}$ after transfection, cells were examined for gene expression, proliferation and cell cycle distribution. For stable transfection, $1 \times 10^{5}$ ECA-109 cells were transfected with the pcDNA3.1/PAQR3 plasmid $(0.5 \mu \mathrm{g})$ and $24 \mathrm{~h}$ later. Subsequent to culturing for another $24 \mathrm{~h}$, the cells were incubated at $37^{\circ} \mathrm{C}$ in DMEM with $15 \%$ FBS containing $600 \mu \mathrm{g} / \mathrm{ml} \mathrm{G} 418$ (Sigma-Aldrich; Merck KGaA) for 3 weeks. The G418-resistant colonies were pooled together and used for colony formation and tumorigenic studies.

$R N A$ extraction and reverse transcriptase-quantitative PCR (RT-qPCR) analysis of tumor specimens. Total RNA was extracted using the TRIzol ${ }^{\circledR}$ (cat. no. 15596018; Invitrogen; Thermo Fisher Scientific, Inc.) according to the manufacturer's one-step instructions. cDNA was synthesized from $3 \mu \mathrm{g}$ total RNA using the M-MLV reverse transcriptase: $4 \mu 15 \mathrm{X}$ buffer, $2 \mu 110 \mathrm{mM}$ dNTPs, $1 \mu \mathrm{l}$ RNA inhibitor and $1 \mu \mathrm{l}$ Reverse transcriptase from the QIAGEN74903-RNeasy Plantmini kit 74903 (cat. no. M1705; Promega Corporation, Madison, WI, USA) according to the manufacturer's instructions. $G A P D H$ was used as the reference gene for RT-qPCR. The forward and reverse primers for PAQR3 were: 5'-TGTCGA AGATGGATGGCATTAGA-3' and 5'-ACCTGACGCCAG TAGTTATTACA-3', respectively. The forward and reverse primers for GAPDH were: 5'-TGTTGCCATCAATGACCC CTT-3' and 5'-CTCCACGACGTACTCAGCG-3', respectively. Gene-specific amplifications of the $20 \mu \mathrm{l}$ PCR mixture were performed using the ABI $7500 \mathrm{HT}$ real-time PCR system (Thermo Fisher Scientific, Inc.). The PCR mixture contained the following: $1 \mu \mathrm{l}$ DNA, 2X SYBR ${ }^{\circledR}$ Select Master mix (cat. no. AT311; Beijing TransGen Biotech Co., Ltd., Beijing, China), and $10 \mu \mathrm{l}$ each of forward and reverse primers $(10 \mu \mathrm{M})$. The PCR amplification conditions were as follows: $95^{\circ} \mathrm{C}$ for $30 \mathrm{sec}, 95^{\circ} \mathrm{C}$ for $60 \mathrm{sec}$ and $72^{\circ} \mathrm{C}$ for $60 \mathrm{sec}$ for a total of 45 cycles, followed by $72^{\circ} \mathrm{C}$ for $7 \mathrm{~min}$. DNA concentration was approximately doubled following each cycle of denaturation, annealing and elongation. The cycle quantification $(\mathrm{Cq})$ of each sample was calculated, and the relative expression of the gene was calculated using the $2^{-\Delta \Delta \mathrm{Cq}}(16)$ method.

Immunohistochemistry. Formalin-fixed paraffin-embedded ESCC tissues (as aforementioned) were sectioned $(4-\mu \mathrm{m})$ for immunohistochemical analysis. For antigen retrieval, the slides were immersed in EDTA [1 mmol/1, $(\mathrm{pH} 8.0)]$ and boiled $60^{\circ} \mathrm{C}$ for $20 \mathrm{~min}$ in a microwave oven. Following rinsing with PBS 3 times, endogenous peroxidase was blocked using $0.3 \%$ hydrogen peroxide for $15 \mathrm{~min}$ at room temperature. The slides were incubated with the PAQR3 primary antibody (1:100; cat. no. ab174327; Santa Cruz Biotechnology, Inc., Dallas, TX, USA), in a humidified chamber at $4^{\circ} \mathrm{C}$ overnight. Following additional washing with PBS three times, the sections were sequentially incubated with horseradish peroxidase-conjugated the anti-rabbit secondary antibody PV-6001 (1:50; Envision ${ }^{\mathrm{TM}}$ detection kit; cat. no. GK500705; Gene Tech Co., Ltd., Hong Kong, China) 

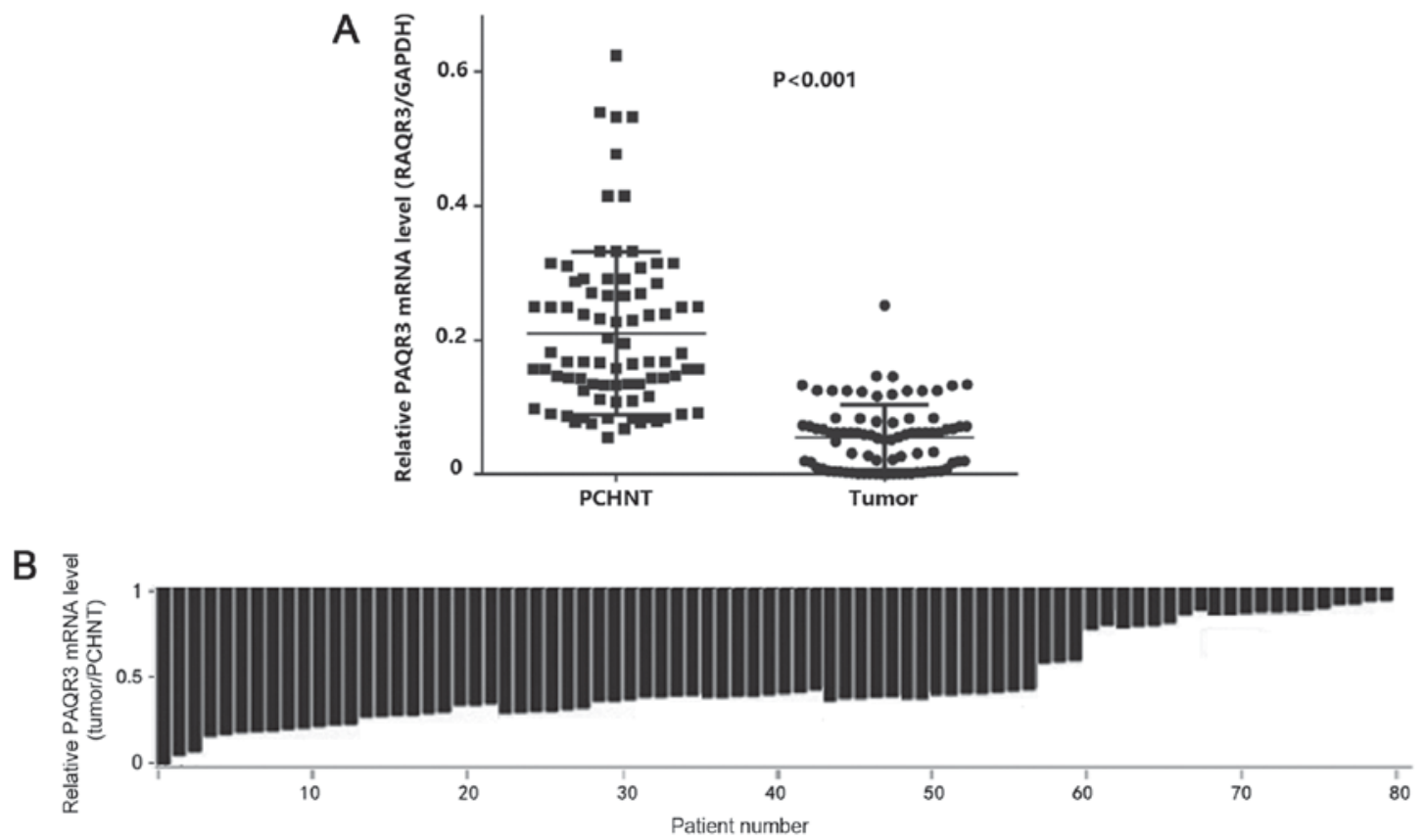

Figure 1. Expression of PAQR3 is reduced in ESCC samples. (A) The relative level of PAQR3 mRNA in human ESCC tissues in comparison with PCHNT specimen from 80 patients with esophageal cancer. PAQR 3 mRNA level was determined by quantitative reverse transcription-quantitative polymerase chain reaction and adjusted for GAPDH. (B) The relative ratio of PAQR3 mRNA in the cancer samples vs. PCHNT samples. PCHNT, paracancerous histological normal tissue; PAQR3, progestin and adipoQ receptor family member 3; ESCC, esophageal squamous cell carcinoma.

at $37^{\circ} \mathrm{C}$ for $30 \mathrm{~min}$, and then washed three times with PBS. Finally, 3,3'-diaminobenzidine tetrahydrochloride was used for signal development and then the sections were lightly counterstained with $20 \%$ hematoxylin at room temperature for $20 \mathrm{~min}$. The paraffin slides were dehydrated (using $95 \%$ ethanol every $2 \mathrm{~h}$ ) and mounted on coverslips. For the negative controls, PBS was used in place of the primary antibody. A light microscope was used at a magnification of $\mathrm{x} 200$. Immunohistochemistry scores of 0-4 were considered to be negative/low expression and 5-11 was considered to be normal/high expression. Anti-PAQR3 (ab174327; Abcam, Cambridge, UK).

Western blot analysis. ECA-109 and TE-1 cells were lysed using radioimmunoprecipitation assay buffer (Beyotime Institute of Biotechnology, Nantong, China) supplemented with protease inhibitors (Sigma-Aldrich; Merck KGaA). Proteins were quantified using a BCA assay. Total protein extract was separated using 5\% sodium dodecyl sulfate-polyacrylamide gel electrophoresis and transferred to nitrocellulose membranes. The membranes were blocked with tris-buffered saline with $10 \%$ Tween-20 three times at room temperature for $60 \mathrm{~min}$. Following incubation at $4^{\circ} \mathrm{C}$ for $24 \mathrm{~h}$ with the primary antibodies [anti-phosphorylated-AKT serine/threonine kinase 1 (p-Akt Ser473; 1:1,000; cat. no. 9271), anti-Akt (1:1,000; cat. no. 9272), and anti- $\beta$-actin antibody (1:500; cat. no. 8457), E-cadherin antibody (1:500; cat. no. 15148), (all from Cell Signaling Technology, Inc., Danvers, MA, USA)], membranes were probed with horseradish peroxidase-conjugated the anti-rabbit secondary antibodies (1:3,000; cat. no. 074-1506; Santa Cruz Biotechnology, Inc.) for $30 \mathrm{~min}$ at room temperature. Signals were visualized using an enhanced chemiluminescent system (Cell Signaling Technology, Inc.) and quantified using Quantity One software v.4.6 (Bio-Rad Laboratories, Inc., Hercules, CA, USA).

Statistical analysis. All statistical analyses were performed using the SPSS 17.0 statistical software (SPSS, Inc., Chicago, IL, USA). Count data were analyzed using the $\chi^{2}$ test and Fisher's exact test. Paired-samples Student's t-test was used to compare mRNA/protein expression of PAQR3 in ESCC tissues with paired adjacent non-tumor tissue samples. Spearman's correlation analysis was used to compare the mRNA/protein expression of PAQR3 in ESCC tissues. Survival analysis was performed using the Kaplan-Meier estimator method, and compared using the log rank test. Prognostic analysis was performed using the Cox proportional hazards regression model. $\mathrm{P}<0.05$ was considered to indicate a statistically significant difference

\section{Results}

PAQR3 mRNA expression is downregulated in ESCC tissue. The levels of PAQR3 mRNA expression in the 80 ESCC and corresponding PCHNT specimens were measured using RT-qPCR. The mRNA expression of PAQR3 was significantly lower in ESCC tissues compared with that in the corresponding PCHNT tissues $(\mathrm{P}<0.0001$; Fig. 1A). A total of $71.25 \%$ of subjects $(57 / 80)$ had significantly lowered PAQR3 mRNA expression in their ESCC tissues compared with the PCHNT tissues (Fig. 1B). Low PAQR3 mRNA expression was defined as ESCC/PCHNT <0.5. All 80 pairs of specimens were independently measured twice.

PAQR3 protein expression is downregulated in ESCC tissue. PAQR3 protein was detected by immunohistochemical 
Table I. Correlations between the PAQR3 expression level and clinicopathological characteristics of 80 cases of ESCC.

\begin{tabular}{|c|c|c|c|c|c|}
\hline \multirow[b]{2}{*}{ Clinicopathological parameters } & \multirow[b]{2}{*}{$\mathrm{N}$} & \multicolumn{2}{|c|}{ PAQR3 mRNA level } & \multirow[b]{2}{*}{$\chi^{2}$} & \multirow[b]{2}{*}{ P-value } \\
\hline & & Low & High/normal & & \\
\hline All cases & 80 & 57 & 23 & & \\
\hline Age, years & & & & 1.807 & 0.369 \\
\hline$<50$ & 37 & 24 & 13 & & \\
\hline$\geq 50$ & 43 & 33 & 10 & & \\
\hline Sex & & & & 0.975 & 0.446 \\
\hline Female & 40 & 27 & 13 & & \\
\hline Male & 40 & 30 & 10 & & \\
\hline Nationality & & & & 3.101 & 0.032 \\
\hline Han & 25 & 15 & 10 & & \\
\hline Kazak & 30 & 23 & 7 & & \\
\hline Uygur & 25 & 19 & 6 & & \\
\hline Tumor length, cm & & & & 5.782 & 0.019 \\
\hline$<5$ & 35 & 19 & 16 & & \\
\hline$\geq 5$ & 45 & 38 & 7 & & \\
\hline Subsection & & & & 0.291 & 1.327 \\
\hline Upper & 24 & 17 & 7 & & \\
\hline Middle & 36 & 24 & 12 & & \\
\hline Lower & 20 & 16 & 4 & & \\
\hline Gross pathologic classification & & & & 0.104 & 2.117 \\
\hline Medullary & 30 & 23 & 7 & & \\
\hline Mushroom & 23 & 16 & 7 & & \\
\hline Ulcer & 17 & 13 & 4 & & \\
\hline Narrowing & 10 & 5 & 5 & & \\
\hline Differentiation & & & & 2.177 & 0.242 \\
\hline Moderate/high & 50 & 36 & 14 & & \\
\hline Poor & 30 & 21 & 9 & & \\
\hline T stage & & & & 1.909 & 0.331 \\
\hline $\mathrm{T} 1 / \mathrm{T} 2$ & 43 & 30 & 13 & & \\
\hline $\mathrm{T} 3 / \mathrm{T} 4$ & 37 & 27 & 10 & & \\
\hline Lymph node metastasis & & & & 7.398 & 0.011 \\
\hline Negative & 38 & 23 & 15 & & \\
\hline Positive & 42 & 34 & 8 & & \\
\hline Recurrence & & & & 9.273 & 0.009 \\
\hline No & 48 & 30 & 18 & & \\
\hline Yes & 32 & 27 & 5 & & \\
\hline Distance metastasis & & & & 0.603 & 0.885 \\
\hline No & 60 & 45 & 15 & & \\
\hline Yes & 20 & 12 & 8 & & \\
\hline
\end{tabular}

PAQR3 mRNA low expression is defined as tumor/PCHNT $<0.5$ and normal/high expression as tumor/PCHNT $\geq 0.5$. P-values are from the Pearson $\chi^{2}$ tests. PAQR3, progestin and adipoQ receptor family member 3; T, tumor.

staining. The normal and adjacent tissues stained positive, in contrast with the staining of PAQR3 in cancer tissues, which was mostly negative (Fig. 2A). PAQR3 protein expression scores were also significantly reduced in EC tissues compared with adjacent normal tissues $(\mathrm{P}<0.0001$; Fig. 2B). The protein levels of PAQR3 were significantly correlated with its mRNA levels ( $\mathrm{r}=0.783$; $\mathrm{P}=0.014$; Fig. 2C).

Association between PAQR3 expression and clinicopathological characteristics of ESCC. The association between 


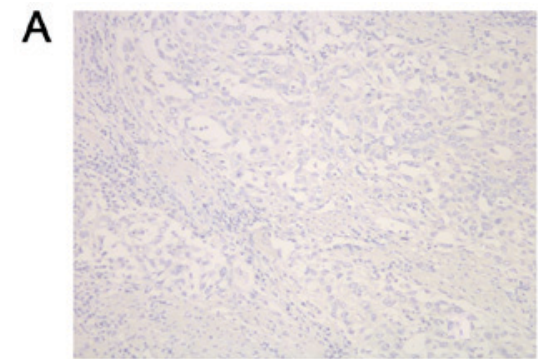

B

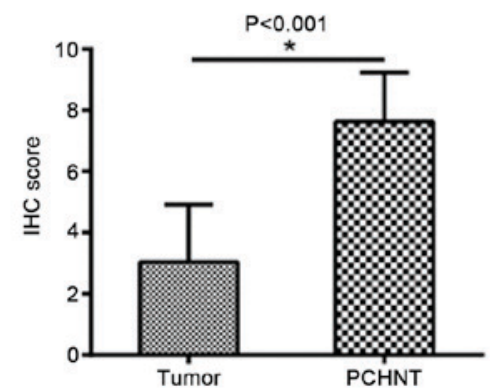

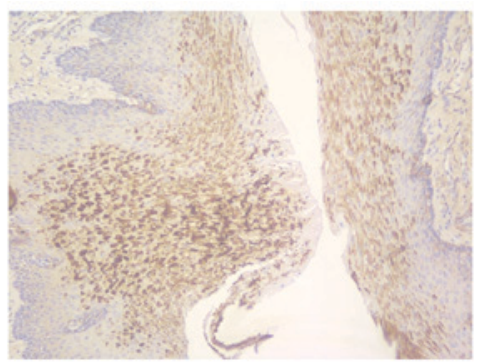

C

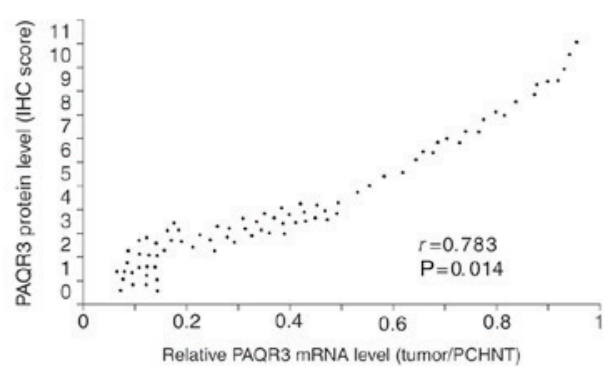

Figure 2. Expression of PAQR3 protein is reduced in ESCC samples. (A) Representative IHC images of PAQR3 expression are shown for normal esophageal tissue, PCHNT and cancer tissues, magnification, x200. (B) The relative ratio of PAQR3 protein in the cancer samples vs. PCHNT samples. (C) The correlation between PAQR3 mRNA level (tumor/PCHNT) as determined by reverse transcriptase-quantitative polymerase chain reaction and PAQR3 protein level as determined by IHC in 80 esophageal cancer tissues. ${ }^{*} \mathrm{P}<0.001$. IHC, immunohistochemistry; PCHNT, paracancerous histological normal tissue; PAQR3, progestin and adipoQ receptor family member 3 .
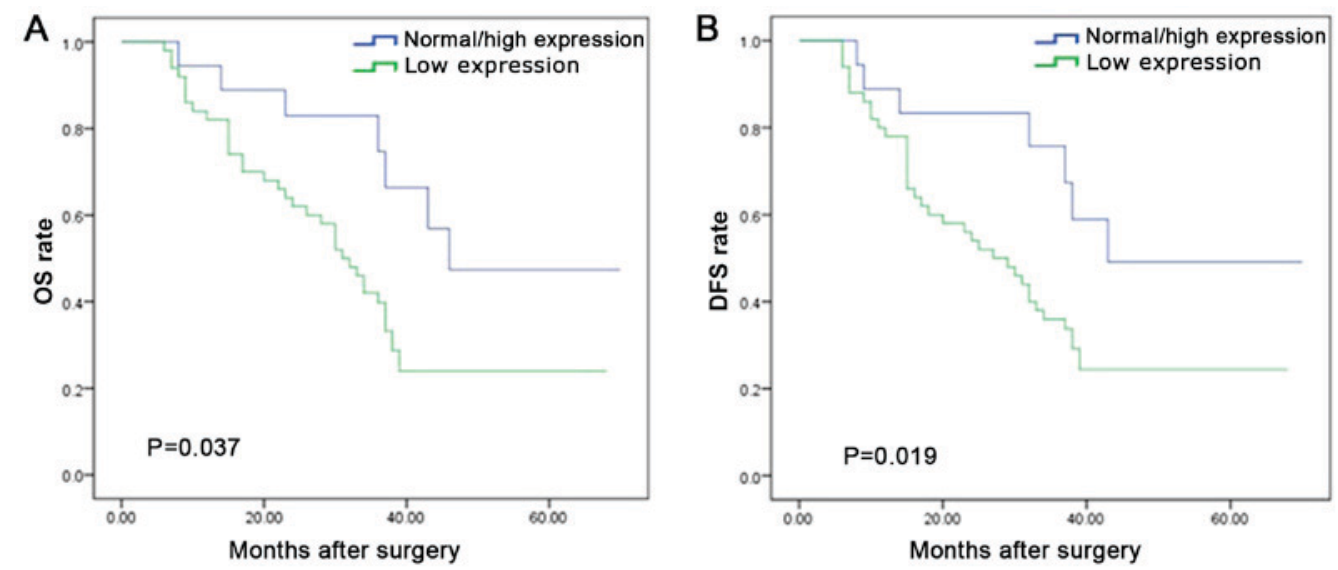

Figure 3. Correlation of PAQR3 expression level with survival of patients with ESCC. Kaplan-Meier curves of (A) OS and (B) DFS in post-surgery patients with ESCC according to the expression level of PAQR3. OS, overall survival; DFS, disease-free survival; PAQR3, progestin and adipoQ receptor family member 3; ESCC, esophageal squamous cell carcinoma.

PAQR3 expression and clinicopathological characteristics of ESCC was examined using the $\chi^{2}$ test. Low PAQR3 expression was significantly associated with ethnic group $(\mathrm{P}=0.032)$, tumor length $(\mathrm{P}=0.019)$, lymph node metastasis $(\mathrm{P}=0.011)$ and local recurrence $(\mathrm{P}=0.009)$. However, $\mathrm{PAQR} 3$ expression was not significantly associated with age $(\mathrm{P}=0.369)$, sex $(\mathrm{P}=0.446)$, tumor location $(\mathrm{P}=1.327)$, tumor type $(\mathrm{P}=2.117)$, tumor differentiation $(\mathrm{P}=0.242)$, $\mathrm{T}$ staging $(\mathrm{P}=0.331)$ or distant metastasis $(\mathrm{P}=0.885$; Table I).

Downregulated PAQR3 expression is associated with ESCC prognosis. In order to evaluate the feasibility of PAQR3 expression as a prognostic factor for ESCC, multivariate survival analysis was performed on all parameters using the Cox proportional hazards regression model. It was determined that OS time was significantly dependent on lymph node metastasis $(\mathrm{P}=0.021)$, local recurrence $(\mathrm{P}=0.036)$ and $\mathrm{PAQR} 3$ expression level ( $\mathrm{P}=0.047$; Table II). OS time was significantly reduced in patients with ESCC and a low PAQR3 expression compared with those with a high PAQR3 expression (median survival, 30 vs. 46 months; $\mathrm{P}=0.037$; Fig. 3A). Furthermore, lymph node metastasis $(\mathrm{P}=0.004)$, local tumor recurrence $(\mathrm{P}=0.029)$ and $\mathrm{PAQR} 3$ expression level $(\mathrm{P}=0.033)$ were independent prognostic factors for the 5-year DFS rate of patients with ESCC (Table II). DFS time was significantly reduced in patients with ESCC and a low PAQR3 expression compared with those with high PAQR3 expression (median survival, 26 vs. 43 months; $\mathrm{P}=0.019$; Fig. 3B). The 1-year, 3-year and 5-year OS rates were as follows: PAQR3 normal/high group, 93, 68 and 45\%; PAQR3 low expression group, 82, 35 and 25\% $\left(\chi^{2}=14.497 ; \mathrm{P}<0.05\right)$. The 1-, 3 - and 5-years DFS rates were as follows: PAQR3 normal/high group, 88, 74 and 50\%; PAQR3 


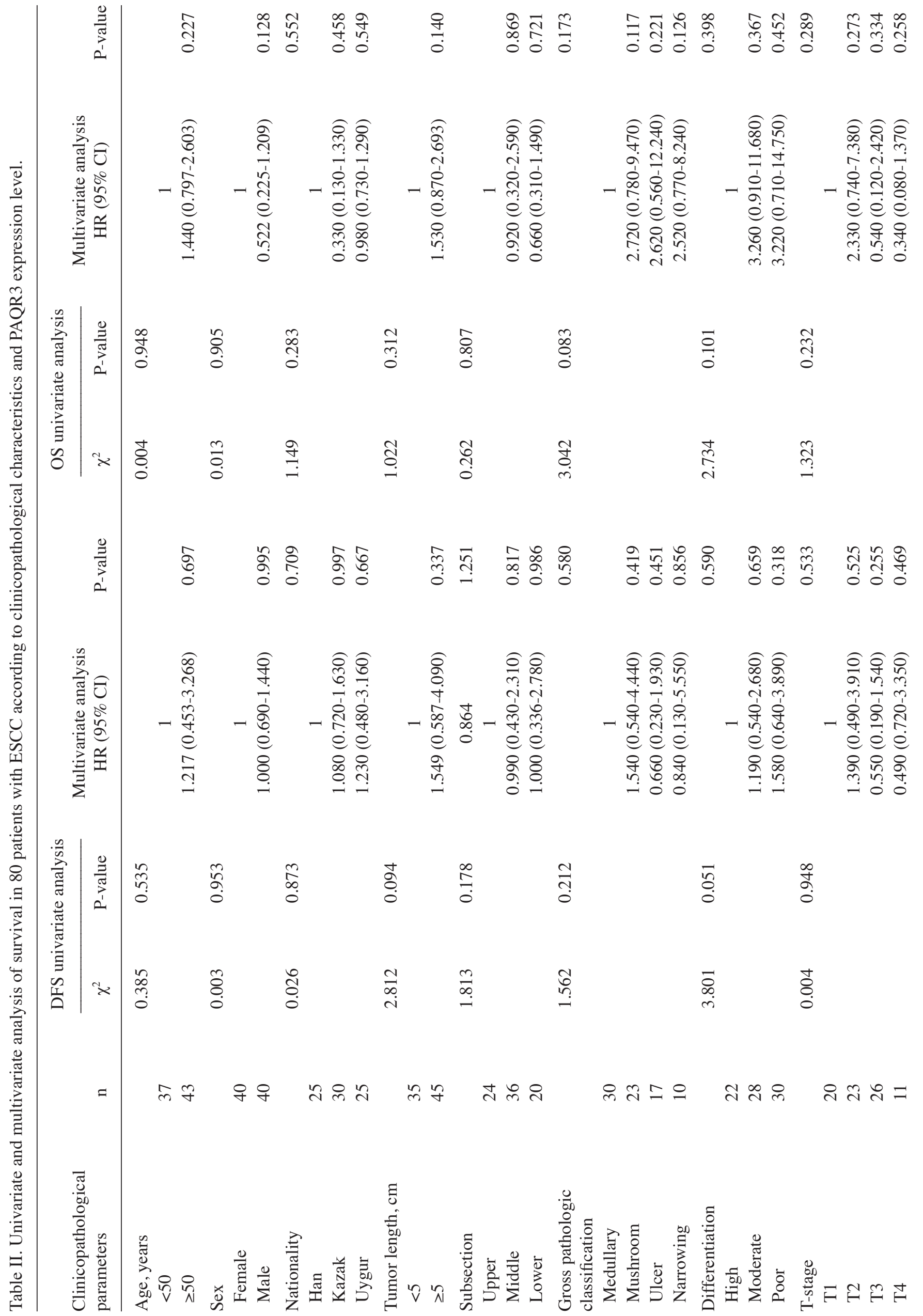




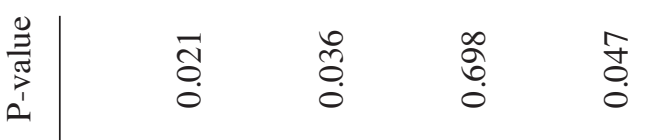

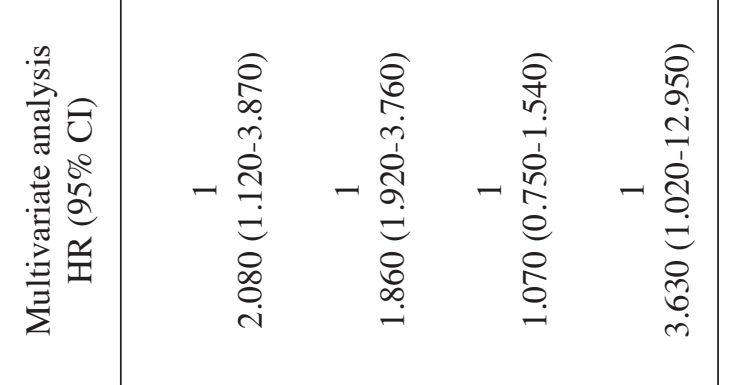

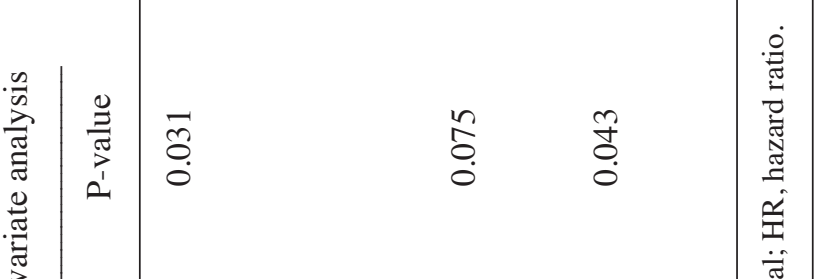

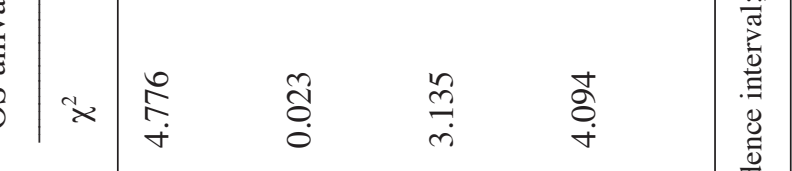

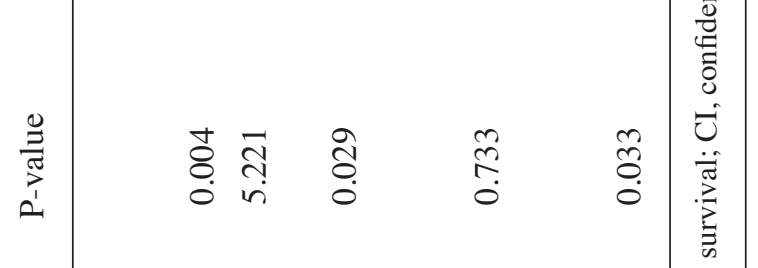

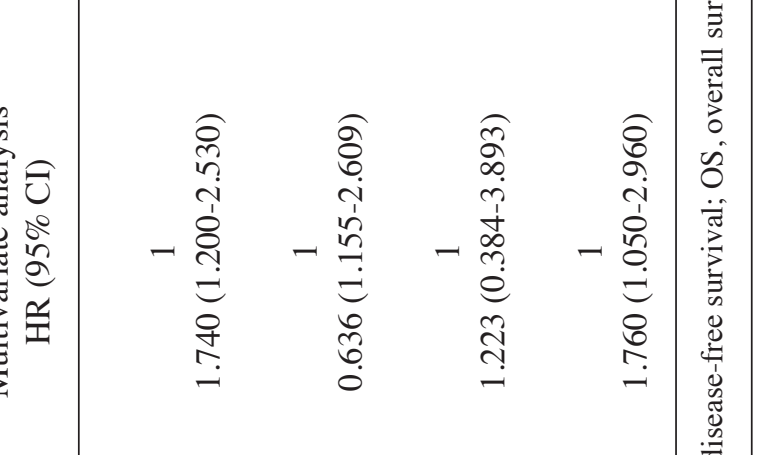

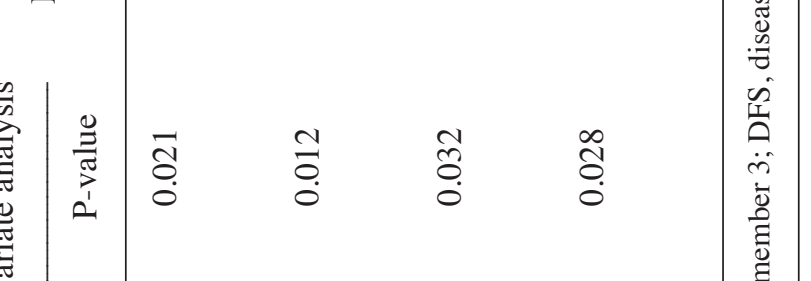


A

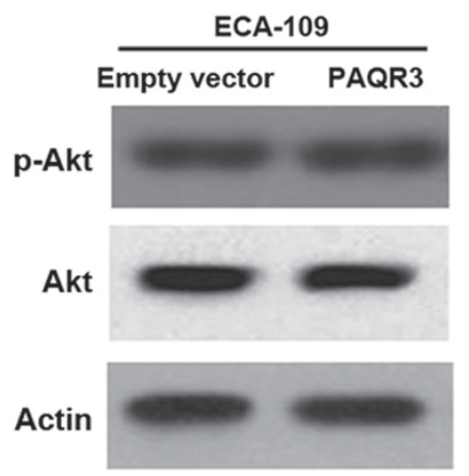

B

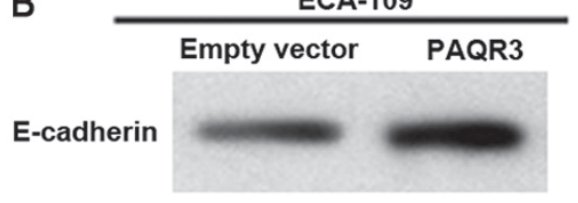

Actin

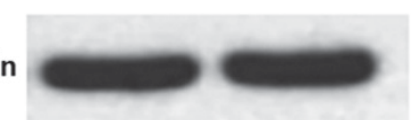

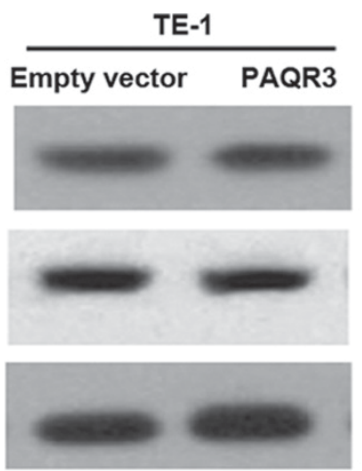

TE-1
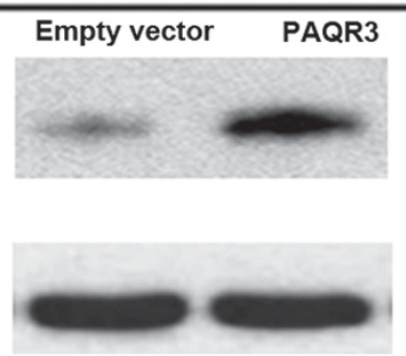

Figure 4. PAQR3 suppresses epithelial-mesenchymal transition features in human esophageal squamous cancer cells. Western blot analysis of indicated proteins in ECA-109 and TE-1 cells transfected with the PAQR3-expressing plasmid or vector $(n=3)$. (A) The phosphorylation of Akt was not altered by PAQR3 overexpression. (B) ECA-109 and TE-1 cells with overexpression PAQR3 were used to analyze the expression of epithelial markers E-cadherin. E-cadherin, epithelial-cadherin; PAQR3, progestin and adipoQ receptor family member 3; p, phosphorylated; Akt, AKT serine/threonine kinase 1.

low expression group: 80,36 and $23 \%\left(\chi^{2}=19.038 ; \mathrm{P}<0.05\right)$ (data not shown).

PAQR3 suppresses epithelial-mesenchymal transition (EMT) features in human ESCC cells. Previous studies have revealed that PAQR3 is able to inhibit Ras/Raf/MEK/ERK signaling cascades. Whether PI3K/AKT signaling pathways were also affected by PAQR3 in ESCC cells was explored (15). Western blot analysis of p-Akt and Akt proteins in ECA-109 and TE-1 cells transfected with the PAQR3-expressing plasmid or vector was performed. However, the activation of Akt was not altered by PAQR3 overexpression (Fig. 4A).

The effect of PAQR3 on EMT was also analyzed, a critical step for tumor migration and metastasis (10). In ECA-109 and TE-1 cells, overexpression of PAQR3 suppressed EMT features as demonstrated by the marked increase in epithelial marker, E-cadherin (Fig. 4B).

\section{Discussion}

PAQR3 is a member of the PAQR family (17). A search of this gene in the world's largest cancer microarray database, Oncomine (https://www.oncomine.org/resource/login.html), demonstrated that PAQR3 is associated with multiple cancer types, including breast cancer, colon cancer, gastric cancer, leukemia and lymphoma. In addition, the expression of PAQR3 mRNA is downregulated in numerous cancer types, including gastric cancer, but upregulated in a number of cancer types of the circulatory system, including leukemia and lymphoma (18). However, there are no relevant data on the expression of PAQR3 in EC. In the present study, the levels of PAQR3 mRNA expression in 80 ESCC and corresponding PCHNT tissue specimens were measured. In addition, a complete follow-up database was constructed to examine the association between PAQR3 and the prognosis and clinicopathological characteristics of patients with ESCC, and to increase the understanding of the diagnosis and prognosis of EC, a highly prevalent disease in Xinjiang, China (19).

RT-qPCR analysis demonstrated that PAQR3 mRNA expression was significantly lower in ESCC tissues compared with in PCHNT tissues, which was consistent with the data in other cancer types, including breast cancer (11), colon cancer (15) and gastric cancer (13), demonstrating that PAQR3 may be an ESCC suppressor gene. The tumor suppressive function of PAQR3 is primarily achieved by inhibiting activation of the ERK signaling pathway (9). The broad anticancer activity of PAQR3 is mediated through the alteration of multiple different signaling pathways (20). PAQR3 overexpression decreased the phosphorylation of ERK1/2 in ESCC cells, without affecting the activation of Akt (21). PAQR3 has demonstrated the ability to interfere with multiple aspects of tumor biology, including proliferation, migration, invasion, and EMT $(22,23)$. In the present study, it was revealed that the overexpression of PAQR3 suppressed EMT features as demonstrated by the marked increase in the epithelial marker E-cadherin.

In the present study, convincing evidence that PAQR3 is a novel genetic marker associated with the progression, proliferation, infiltration and prognosis of EC has been indicated. Analysis of the clinicopathological characteristics of 80 patients with EC indicated that the level of PAQR3 mRNA expression was associated with ethnic group, status of lymph node metastasis and tumor length. In particular, the level of PAQR3 mRNA expression was significantly higher among Han patients with ESCC than among those of the Kazakh and Uygur ethnic groups. Although current studies have indicated improved short-term 
efficacy of treatment in Kazakh and Uygur patients with ESCC, their prognosis and long-term survival were lower compared with those of Han patients. Whether this characteristic is associated with the difference in PAQR3 mRNA expression between ethnic groups will require further studies to confirm. In the present study, it was demonstrated that an association between patients with low PAQR3 expression, and lymph node metastasis and poor prognosis, indicating that PAQR3 may be a potential tumor suppressor. The total of 80 histopathological specimens was a limiting factor for this study as the number of samples after grouping was too small, reducing the statistical reliability. In the future study, the sample size should be increased to confirm the credibility of the findings of the present study.

The present study demonstrated that low PAQR3 expression was associated with lymph node metastasis and survival. Multivariate prognostic analysis of the association between PAQR3 expression and the status of lymph node metastasis indicated reduced DFS in patients with low PAQR3 expression and lymph node metastasis. Thus, lymph node metastasis or low PAQR3 expression may be adverse prognostic factors for EC. The combined evaluation of PAQR3 expression and lymph node status may be helpful in the prognosis of patients, and may provide insights into postoperative adjuvant therapy strategies. Notably, a previous study have demonstrated that PAQR3 expression may be associated with the susceptibility of breast cancer to epirubicin, whereby PAQR3 overexpression enhances the susceptibility of breast cancer cells to epirubicin via caspase-associated signaling pathways and epirubicin inhibition-induced ERK activation (24). This data indicated that PAQR3 may be a novel cancer treatment target that can be used to evaluate radiation therapy and chemotherapy sensitivity. However, further studies are required to determine the effect of PAQR3 on the sensitivity of ESCC to treatment.

In conclusion, the present study demonstrated that PAQR3 downregulation is associated with the progression of tumor recurrence and increased lymph node metastasis in EC, and is significantly associated with reduced survival of patients with EC. This data demonstrated that PAQR3 is a tumor suppressor gene associated with the survival and prognosis of esophageal malignancies. PARQR3 may be a potential valuable prognostic factor for ESCC, and a novel predictor of radiation and chemotherapy sensitivity. To the best of our knowledge, the present study is the first to examine the association between PAQR3 and ESCC. However, further in vitro and in vivo studies are required to elucidate the molecular mechanism underlying PAQR3 in the regulation of ESCC.

\section{Acknowledgements}

The present study was supported by the State Key Lab Incubation Base of Xinjiang Major Diseases Research (grant no. SKLIB-XJMDR-2016-5).

\section{Competing interests}

The authors declare that they have no competing interests.

\section{References}

1. Torre LA, Bray F, Siegel RL, Ferlay J, Lortet-Tieulent J and Jemal A Global cancer statistics, 2012. CA Cancer J Clin 65: 87-108, 2015.
2. Kamangar F,Dores GM and Anderson WF: Patterns of cancer incidence, mortality, and prevalence across five continents: Defining priorities to reduce cancer disparities in different geographic regions of the world. J Clin Oncol 24: 2137-2150, 2006.

3. Ferlay J, Shin HR, Bray F, Forman D, Mathers C and Parkin DM: Estimates of worldwide burden of cancer in 2008: GLOBOCAN 2008. Int J Cancer 127: 2893-2917, 2010.

4. Samatar AA and Poulikakos PI: Targeting RAS-ERK signalling in cancer: Promises and challenges. Nat Rev Drug Discov 13: 928-942, 2014

5. Tasioudi KE, Saetta AA, Sakellariou S, Levidou G Michalopoulos NV, Theodorou D, Patsouris E and Korkolopoulou P: pERK activation in esophageal carcinomas: Clinicopathological associations. Pathol Res Pract 208: 398-404, 2012.

6. Feng L, Xie X, Ding Q, Luo X, He J, Fan F, Liu W, Wang Z and Chen Y: Spatial regulation of Raf kinase signaling by PAQR3. Proc Natl Acad Sci USA 104: 14348-14353, 2007.

7. Yu X, Li Z, Chan MT and Wu WK: PAQR3: A novel tumor suppressor gene. Am J Cancer Res 5: 2562-2568, 2015.

8. Fan F, Feng L, He J, Wang X, Jiang X, Zhang Y, Wang Z and Chen Y: PAQR3 sequesters B-Raf to the Golgi apparatus and inhibits the proliferation and tumorigenicity of human malignant melanoma cells. Carcinogenesis 29: 1157-1163, 2008.

9. Xie X, Zhang Y, Jiang Y, Liu W, Ma H, Wang Z and Chen Y: Suppressive function of PAQR3 on chemical carcinogen-induced skin carcinogenesis in mouse. Carcinogenesis 29: 1632-1638, 2008.

10. Wu HG, Zhang WJ, Ding Q, Peng G, Zou ZW, Liu T, Cao RB, Fei SJ, Li PC, Yang KY, et al: Identification of PAQR3 as a new candidate tumor suppressor in hepatocellular carcinoma. Oncol Rep 32: 2687-2695, 2014.

11. Li Z, Ling ZQ, Guo W, Lu XX, Pan Y, Wang Z and Chen Y: PAQR3 expression is downregulated in human breast cancers and correlated with HER2 expression. Oncotarget 6: 12357-12368, 2015.

12. Wang L, Wang X, Li Z, Xia T, Zhu L, Liu B, Zhang Y, Xiao F, Pan Y, Liu Y, et al: PAQR3 has modulatory roles in obesity, energy metabolism, and leptin signaling. Endocrinology 154: 4525-4535, 2013

13. Qiao S, Guo W, Liao L, Wang L, Wang Z, Zhang R, Xu D, Zhang Y, Pan Y, Wang Z and Chen Y: DDB2 is involved in ubiquitination and degradation of PAQR3 and regulates tumorigenesis of gastric cancer cells. Biochem J 469: 469-480, 2015.

14. Ling ZQ, Guo W, Lu XX, Zhu X, Hong LL, Wang Z, Wang Z and Chen Y: A Golgi-specific protein PAQR3 is closely associated with the progression, metastasis and prognosis of human gastric cancers. Ann Oncol 25: 1363-1372, 2014

15. Wang X, Li X, Fan F, Jiao S, Wang L, Zhu L, Pan Y, Wu G, Ling ZQ, Fang J and Chen Y: PAQR3 plays a suppressive role in the tumorigenesis of colorectal cancers. Carcinogenesis 33: 2228-2235, 2012.

16. Livak KJ and Schmittgen TD: Analysis of relative gene expression data using real-time quantitative PCR and the 2(-Delta Delta C(T)) method. Methods 25: 402-408, 2001.

17. Guo W, You X, Xu D, Zhang Y, Wang Z, Man K, Wang Z and Chen Y: PAQR3 enhances Twist1 degradation to suppress epithelial-mesenchymal transition and metastasis of gastric cancer cells. Carcinogenesis 37: 397-407, 2016.

18. Oncomine database (https://www.oncomine.org/resource/login. html).

19. Zhang Y: Xinjiang esophageal cancer distribution. Xinjiang Medical College 11: 139-144, 1988.

20. Wang L, Pan Y, Huang M, You X, Guo F and Chen Y: PAQR3 augments amino acid deprivation-induced autophagy by inhibiting mTORC1 signaling. Cell Signal 33: 98-106, 2017.

21. Bai G, Chu J, Eli M, Bao Y and Wen H: PAQR3 overexpression suppresses the aggressive phenotype of esophageal squamous cell carcinoma cells through the ERK pathway, Biomed Pharmacother 94: 813-819, 2017.

22. Wu Q, Zhuang K and Li H: PAQR3 plays a suppressive role in laryngeal squamous cell carcinoma. Tumour Biol 37: 561-565, 2016.

23. Huang W, Guo W, You X, Pan Y, Dong Z, Jia G, Yang C and Chen Y: PAQR3 suppresses the proliferation, migration and tumorigenicity of human prostate cancer cells. Oncotarget 8: 53948-53958, 2016.

24. Huang J, Xiang T, Luo X, Huang J, Xiao Y, Yang B, Yin X, Li H, Xia Li X, Peng W, et al: RKTG, a RAS/RAF/ERK signaling antagonist regulated by HER2, suppresses malignant phenotypes and enhances chemosensitivity in breast cancer cells. J Third Military Med Univ 8: 1658-1662, 2013. International (CC BY-NC-ND 4.0) License. 